Recepción: 22 / 03 / 2018

Aceptación: 11 / 07 / 2018

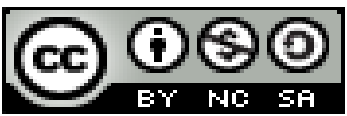

Ciencias económicas y empresariales

Publicación: 01/ 08/ 2018

Artículo de investigación

\title{
Vinculación entre la universidad, Estado y empresa para el desarrollo económico de Ecuador
}

Linkage between the university, State and company for the economic development of Ecuador

\section{Articulação entre universidade, Estado e empresa para o desenvolvimento econômico do Equador}

\author{
Angélica E. Borja-Arévalo ${ }^{\mathrm{I}}$ \\ angelica.borjaa@ug.edu.ec \\ Ana J. Haro-Velasteguí II \\ ana.harov@ug.edu.ec \\ Pedro A. Huacón-Cru ${ }^{\text {III }}$ \\ pedro.huaconc@ug.edu.ec
}

\section{Correspondencia: jgamboap@unemi.edu.ec}

\footnotetext{
I'Magister en tributación, Ingeniera comercial, Universidad de Guayaquil, Guayaquil, Ecuador.

II Magister en Ciencias Internacionales y Diplomacia, Ingeniera en Marketing y Negociación Comercial, Universidad de Guayaquil, Guayaquil, Ecuador.

III Magister en administración de Empresas, Contador público autorizado, Universidad de Guayaquil, Guayaquil, Ecuador.
} 


\section{Resumen}

El escenario económico actual y sus evidentes resultados aquejan de modo directo y como resultado ineludible al crecimiento financiero, acarreando graves consecuencias colaterales sociales, culturales y políticas. La investigación fundamentada en documentos, destacadamente bibliográficos, fue la utilizada para el desarrollo de este artículo que tiene como objetivo demostrar la interacción entre la universidad, el Estado y la empresa, como motor imprescindible del desarrollo económico del País. La Universidad interviene como un ente de generación y transferencia del conocimiento, desarrollando la investigación científica y que adicionalmente escudriña la industria, para que sea en ella donde se ejecuten los resultados de dichos descubrimientos, fomentando de manera técnica la innovación en servicios, bienes, y mercancías ya existentes, así como la creación de nuevos productos. El Estado interviene como una entidad inversionista que direcciona los recursos con el objetivo de obtener una rentabilidad social y cultural, afianzando su política de gobierno, fortaleciendo los índices de competitividad y como último eje, la Empresa, como beneficiaria de estos productos debido a que podrá contar con información actualizada y exacta para implementarla ya sea en la tecnificación salvaguardista de la producción o en la creación de nuevos planes de negocios, como fruto de esta integración se obtendrán resultados óptimos como la fomentación fortalecida del emprendimiento.

Palabras clave: universidad; Estado; empresa; conocimiento; inversionista; beneficiaria.

\section{Abstract}

The current economic scenario and its obvious results affect in a direct way and as a result of unavoidable financial growth, causing serious social, cultural and political collateral consequences. This research was based on documents, notably bibliographic, which aims to demonstrate the indispensable and objective interaction between the University, the State and the Enterprise, as an essential engine of the economic development of the country. The University acts as an entity of the generation and transfer of knowledge, developing scientific researching and that additionally squint industry, to make it in it where running the results of these discoveries, encouraging technical innovation in already existing goods, merchandises, and services, as well as the creation of new products; The State intervenes as an investor entity that directs resources in order to achieve social and cultural profitability strengthening the 
Governmental Policy, strengthening the competitiveness rates and as the last axis, the Enterprise, as a beneficiary of these products due to the fact that you can count on up-to-date and accurate information to implement it either in the safeguard modernization production or in the creation of new business plans, as a result of this integration, optimal results as well as the strengthened promotion of entrepreneurship will be obtained.

Keywords: university; state; enterprise; knowledge; investor and beneficiary.

\section{Resumo}

O cenário econômico atual e seus resultados óbvios afetam direta e inevitavelmente o crescimento financeiro, causando sérias consequências colaterais políticas, culturais e sociais. Pesquisa com base em documentos, bibliográfica excepcionalmente, foi utilizada para o desenvolvimento deste artigo tem como objetivo demonstrar a interação entre a Universidade, o Estado ea Sociedade, como um motor essencial do desenvolvimento económico do país. Universidade intervém como uma geração entidade e transferência de conhecimentos, o desenvolvimento de pesquisa científica e examina ainda mais a indústria para estar nele onde os resultados desses achados são implementados, promovendo tecnicamente inovação em serviços, bens e produtos existentes, bem como a criação de novos produtos. O Estado intervém como uma entidade de investimento que direciona recursos a fim de obter uma rentabilidade social e cultural, reforçando a sua política do governo, fortalecendo índices de competitividade com a última eixo, a Companhia, como beneficiário desses produtos, porque eles podem ter informações atualizadas e precisas para implementar tanto a modernização salvaguardista de produção ou criação de novos planos de negócios, como resultado dessa integração como reforçou fomento ideal de resultados de empreendedorismo será obtida.

Palavras chave: universidade; estado; empresa; conhecimento; investidor beneficiário

\section{Introducción}

La situación económica que ha atravesado el país en las últimas décadas, han sido extremistas, debido a que siempre se ha sometido a una mala combinación entre decisiones políticas fiscalesmonetarias y pésimas administraciones gubernamentales de turno, que han dado resultados caóticos, como consecuencia nuestra economía se ha visto muy vulnerable y susceptible a cualquier cambio que en el mercado mundial pueda ocurrir, debido a la globalización, que lo 
podemos definir como un proceso; social, cultural, tecnológico y económico, que a través de una adecuada e inmediata comunicación creciente a nivel mundial unen los mercados, consolidando así sociedades y culturas, considerando también como un proceso dinámico, en resumen este fenómeno provoca que vez un país se pueda evidenciar fácilmente la crisis, en tanto y cuando no se tomen las medidas adecuadas, como resultado podemos evidenciar graves consecuencia colaterales sociales, culturales y políticas.

Como solución inminente el actual gobierno, está fomentando una interacción indispensable y objetiva entre la Universidad, el Estado y la Empresa, como motor imprescindible del desarrollo económico del País, cuyo objetivo de esta triangulación se concentra en fortalecer el emprendimiento a niveles de competitividad canalizado adecuadamente los conocimientos técnicos y científicos.

En la cual la universidad juega un rol muy importante, debido a que se centrará como un ente de generación y transferencia del conocimiento ( hacia los diferentes agentes que intervienen en el mercado), desarrollando la investigación científica (en función a la información que fluye de los diferentes procesos), y que adicionalmente escudriña la industria, para que sea en ella donde se ejecuten los resultados de dichos descubrimientos, fomentando de manera técnica la innovación en servicios, bienes, y mercancías ya existentes, así como la creación de nuevos productos en conjunto con la aplicación de la tecnología que actualmente contamos.

Por su parte, el Estado interviene como una entidad inversionista que direcciona los recursos con el objetivo de obtener una rentabilidad social y cultural, afianzando su política de gobierno, fortaleciendo los índices de competitividad y como último eje, en otras palabras el estado interviene como un socio estratégico que busca como único fin un resultado social, el cual involucra e intervienen de manera inherentes los objetivos del Plan Nacional del Buen Vivir (PNBV), los cuales son el resultado del Plan de Gobierno que en conjunto con la Matriz Productiva, coadyuvan a fortalecer en parte u nuestra vulnerable economía.

Como último eje de esta triangulación, tenemos La Empresa como beneficiaria de estos productos, debido a que podrá contar con información actualizada y exacta para implementarla ya sea en la tecnificación salvaguardista de la producción o en la creación de nuevos planes de negocios (reingeniería de procesos), como fruto de esta integración se obtendrán resultados óptimos como la fomentación fortalecida del emprendimiento los cuales estarán canalizados en los sectores estratégicos que se están proponen actualmente en el mercado. 
Las Universidades y El Emprendimiento Bajo la Óptica de Otros Investigadores

Pineda \& Márquez (2011) presentaron su revisión de literatura en torno a los más destacados modelos y mecanismos de interacción Universidad-país-entorno, cuya finalidad era identificar de entre ellos retos que lleven a mejoras a la universidad colombiana.

Velho, \& Velho (2010) describieron y analizaron las políticas, así como el plan de acción llevado a cabo por los países pertenecientes al grupo MERCOSUR con el objeto de promocionar el vínculo entre los motores principales que mueven las economías de los países gobiernouniversidad-empresas, demostrando que los modelos, políticas e instrumentos utilizados en los países industrializados para motivar este vínculo fueron muy pronto también utilizados por países en desarrollo puntualmente señalaron a los que son miembros del MERCOSUR.

Ramírez (2010) luego de investigar determinaron que, con el objetivo de originar otras formas de emprendimiento para los sectores económicos de cada región colombiana, este país ha puesta la mira en las sinergias y en la aplicación de experiencias de éxito de las dinámicas observadas y comprobadas en otros países. El gobierno de Colombia tiene el reto de apoyar la fundación y consolidación de comités Universidad-Empresa-Estado, que surgen tras políticas de ciencia, innovación y tecnología, que han logrado la predisposición del mundo empresarial y hacer puente con la academia.

\section{El Emprendimiento en el Ecuador y su Evolución.}

Uno de los factores que incentivan en primer lugar a una persona en emprender, es precisamente la autonomía, es una agradable sensación de jerarquía que empodera al individuo, convirtiéndose muchas veces a un modelo a seguir (para a aquellos que se han mantenido en el mercado), pero en efecto negativo, son para aquellos que fracasan, los cuales se pueden atribuir a ciertos factores negativos (tanto endógenos como exógenos), en segundo lugar se encuentra los réditos que puedan obtener como resultado del emprendimiento, como derivado de estos podemos concluir, que el objetivo general es buscar el bienestar y el status que buscan para la familia, garantizando una seguridad económica y social.

El Estado con el afán de garantizar el bienestar de la ciudadanía, la cual consiste en garantizar la vida digna la cual requiere accesos universal y permanente a bienes superiores, así como la promoción del ambiente adecuado para alcanzar las metas personales y colectivas (Plan Nacional del Buen Vivir, Objetivo no3), con esta primicia, el estado como órgano rector desea a través de 
sus diferentes programas desarrollar estrategia que puedan mitigar cualquier variable que afecten el bienestar de los ciudadanos.

Muchas de las debilidades que el emprendedor se pueden enfrentar es que cuenta solamente con conocimientos empíricos y semi-técnicos, el conocimiento empírico lo podemos definir, como aquel basado en experiencia, determinado en la percepción de lo que existe y sus características específicas, y los conocimientos semi-técnicos, que por su definición se enfoca, a la noción referente a un procedimiento que sigue un objetivo para un determinado fin o resultado, aparentemente estos podrían ser dos factores primordiales para poder obtener un óptimo resultado en cualquier emprendimiento, no obstante lo que se necesita es una educación integral del emprendedor para lo cual debe de tener acceso al conocimiento y herramientas necesarias para poder a solventar cualquier inconveniente que se le llegase a presentar.

En la actualidad Ecuador cuenta con una alta tasa de emprendimiento que lo ubica entre los más altos de región, durante los años 2013 y 2014 asciende a 36\% y 32,6\% respectivamente, para poder entender este esta evolución existe una varios criterios de valores que se analizó, entre los principales podemos destacar la oportunidad/Necesidad, que es un indicador que muestra y sustenta la realidad ecuatoriana del porque emprender, otro indicadores que se considera según el estudio Global Entrepreneurship Monitor (GEM), es el motivacional que se enfoca en aquellos emprendedores dobles, que poseen ya un negocio establecido aquellos que poseyendo un negocio nuevo, están en proceso de crear otro y por lo tanto son nuevos y nacientes a la vez (Izquiero, 2014).

La universidad como eje integral conocimiento Chaverri (2012, p. xxx), plantea que las universidades son las portadoras y generadoras del conocimiento como su recurso primordial, elemento con "el que cuenta la sociedad para desarrollarse integralmente"; esta aseveración convierte a las universidades en las principales responsables del escenario social actual. Las universidades ofrecen un servicio que en realidad son armas poderosas, mismas que debidamente utilizadas llegarían a obtener como resultado la toma de la decisión de "ganar-ganar" en la sociedad. Una sociedad involucrada en el aprendizaje que la academia ofrece sometería por ejemplo el problema de la inseguridad actual a un análisis consciente de la que muy probablemente generaría nuevas fuentes de trabajo. 
La Universidad como ente generadora de conocimiento tiene el fiel compromiso de actuar de manera integral en el desarrollo de los individuos ante la sociedad, siendo los emprendedores su gran interés, para lo cual le facilitara todas las herramientas necesarias, la universidad como centro educativo se ha venido reestructurando su misión acorde a las necesidades de la sociedad, con el propósito de cumplir con el propósito del gobierno en el cual garantiza el fortalecimiento de la capacidades y potencialidades de la ciudadanía a través de la transferencia de conocimientos y vinculación con la sociedad, mitigando de esta manera el analfabetismo (Plan nacional del Buen Vivir Objetivo No.4).

Actualmente, la universidad lo busca es abrir campo en el aspecto social y económico, a través de implementación de una coordinada y asertiva estratégica de transferencia de conocimiento, para el cual se está organizando reuniones de trabajo arduo con las respectivas comisiones que buscan como objetivo la adecuada integración de saberes con las prácticas y la debida vinculación con la sociedad.

\section{El Estado y su relación con el emprendimiento}

Minniti (2012, p. XXX), por medio de un exhaustivo análisis de las evidencias generadas por investigaciones recientes, esta articulista sostiene que no puede haber crecimiento económico sostenido en un país sin emprendimiento. "No obstante, ambos solamente funcionan gracias a la labor de intermediación y a la asunción de riesgo de las actividades llevadas a cabo por los emprendedores". Ambos actúan como colegas cuando de la actividad económica se trata "transformando las oportunidades de beneficio no explotadas en productos y servicios comercializables"

El estado por su parte, como ente regulador pone a disposición todas las opciones necesarias (programas gubernamentales), para poder mantener y sostener los proyectos de innovación, en los últimos años el país preocupado por este tema ha venido trabajo de manera ardua y pre cautelosa cada cambio integral de las políticas y procedimientos, fue sustentado acorde del Plan Nacional del Buen Vivir y la transformación de la matriz productiva, cuyo objetivo esa enfocado en la innovación y competitividad, los cuales para poder lograrlo se está trabajando en el valor agregado en la elaboración del producto y/o servicio, como segundo paso el estado busca la sustitución de las importaciones por los productos locales. 
La empresa como beneficiaria de los resultados.

La empresa como último eje de la piedra angular de esta triangulación, es la beneficiaria de los resultados, de los productos como investigaciones científicas que como consecuencia se ve reflejado en la reingeniería y el perfeccionamiento de los procesos, de esta manera podríamos obtener productos de calidad y generar un nivel de confianza en el individuo que busca la excelencia al momento de consumir, este apartado va de la mano con lo que desea de manera idónea para la producción del estado, que consiste la fomentación de la transformación de la matriz productiva, cumpliendo así con este objetivo la equidad y solidaridad en lo que respecta a los conocimientos (Plan nacional del Buen Vivir Objetivo 10).

\section{Capital Semilla, en América Latina}

Uno de los grandes obstáculos que poseen el emprendedor latinoamericano es justamente la restricción a la fuente de financiamiento, provocando así el poco desarrollo de empresas innovadoras, como resultado del mismo tenemos un poco crecimiento de la economía, el contraste lo podemos observar en países desarrollados como: Estados Unidos, Canadá, Australia e Irlanda, que estimulan las creaciones de empresas innovadoras y fomentan el alto crecimiento de la economía.

La diferencia estos países, consiste en la tecnificación que diseñan, implementan y controlan al momento de la evaluación de los proyectos (considerando la adecuada administración de recursos tecnológicos y financieros), y posteriormente proceden a financiar estas empresas que por lo general son jóvenes emprendedores, como resultado de este proceso obtenemos alta rentabilidad y con riesgos diversos que puedan tener el desarrollo en el mercado, todo eso se refleja en el plan de negocio adecuadamente estructurado, adicionalmente existen redes de empresas que tienen como objetivo primordial financiar proyectos rentables que un corto plazo genere altos réditos, otro factor que sustenta este procesos son variables de entornos inherentes tales como: legales y cultural.

En América Latina, países como Argentina, Chile, Perú y El Salvador, son economías que en las últimas décadas han emergido, presentando una estabilidad económica, no obstante el factor que respalda esta primicia es que su matriz productiva está direccionada en los recursos naturales, que por su concepto puede ser susceptible a los cambios vulnerable que se puedan presentar en el 
mercado, pero debido a su escases lo vuelven único, siendo Chile y Colombia pionero en la producción de recursos naturales (minerales).

Una opción acertada que se ha implementado para sustentar el adecuado mecanismo es la creación de centros especializados emprendedores, que a través de adecuadas opciones que incluyen el proceso de la evolución de las empresas, así como foros físicos, virtuales y clubes e institutos que busca la educación de los emprendedores, cumpliendo el objetivo primordial que consiste un adecuado flujo y poder preparar adecuadamente a los emprendedores.

\section{América Latina y apoyo público}

Como parte de coadyuvar a las falencias que han tenido los fracasos de los emprendimientos, el gobierno a creados programas que cierta manera busca es perfeccionar estas fallas a través de perfeccionar los canales de comunicaciones que ayuden al emprendedor a formular adecuadamente sus proyectos desde la concepción de las ideas hasta el desarrollo como tal de la empresa, en otras palabras, educar, facilitarles privilegios que le puedan determinar y poder llegar al objetivo deseado.

Todo esto conlleva a crear incentivos en el sector financiero y de esta manera creer en él, los emprendimientos que en primera estancia se forman PyMES, se han creado Fideicomiso Financiero, que tienen como objetivo, otorgar financiamiento a corto y mediano plazo, a proyecto en expansión, en otras palabras son los recursos son orientado a PyMES que requieran realizar una reingeniería que prolongue el sustento del proyecto, que puede estar basado en la creación de productos innovadores o la extensión de algún productos que se deseen desarrollar.

El segmento objetivo es financiero aquellos proyectos que se enfoquen o estén direccionados la creación de productos que sustituyan las importaciones, combinando con un alto impacto en el desarrollo regional, esto quiere decir incentivar el producto nacional y ser los productores primarios y evitar la re-exportación de la materia prima, dinámica que es muy común en nuestros países regionales.

Con estos mecánicos, en la actualidad podemos visualizar que existe una buena aceptación por los inversionistas privados, que están canalizando los recursos a estos proyectos. 
Entes gubernamentales preocupados por el desarrollo de estos proyectos, incentivan a los emprendedores, con cantidades significativas, que son otorgados, pero con la condición que las incubadoras actúen como vigilantes y supervisores de todo el proceso del emprendimiento.

Otro factor indispensable, se enfocan es la innovación y la tecnología, ambos factores son indispensables al momento de poder evaluar un proyecto, todo esto se debe a un gran interés del gobierno de generar productos no comunes, que ayude a la realizar una reingeniería local para poder igualarse con la evolución del mercado de los países desarrollados, en combinación con inversión privada da como resultado el crecimiento mutuo y podrían ser socios estratégicos.

El cambio en la gestión empresarial ante la globalización internacional.

En la actualidad podemos ver que, en el mercado, existe un cambio radical en el ámbito tecnológico y organizativo empresarial, con la globalización y la tecno-globalización (considerando lo tecnológico como innovación), que habrá de mantenerse en un futuro previsible. Existe un efecto directamente proporcional entre las ventajas competitivas y la economía en crecimiento que consiste en los bajos costos laborales, para poder trabajar en un ritmo de trabajo más alto, esto quiere decir la diversificación e incremento tecnológico, viéndose reflejando en los últimos años, las nuevas teorías del crecimiento económico se basan sobre el hecho de que las fuerzas para un desarrollo sostenible son el incremento de los conocimientos y los cambios tecnológicos y no en la tradicional acumulación pura y simple de capitales o de maquinaria y equipo de alto contenido tecnológico. Las innovaciones están también acelerando la obsolescencia de los conocimientos y la experticia, todo esto conlleva a la creación del conocimiento y la necesidad imperante de crear nuevas estrategias que simplifiquen la dinámica de los proyectos de emprendimiento.

\section{Para poder determinar las principales características que se encuentran en esta nueva era} de la economía del conocimiento, podemos citar lo que describen algunos autores como sigue:

Desmaterialización en su libro "The Intelligent Enterprise" el profesor James Brian Queen señala que el conocimiento se ha convertido en las tres cuartas partes del valor agregado en el sector manufacturero 
Conectividad, Don Tapscott y Arc Caston en su libro "Paradigm Shift. The Promise of the Information Technology" han identificado una serie de discontinuidades entre las máquinas y la era de la información, lo cual ha conducido a una reestructuración de las economías nacionales para formar sectores productivos más orientados a la creación, uso y difusión del conocimiento Redes virtuales, también Don Tapscott en su libro "Digital Economy. The Promise and Perfil in the Age o Networked Intelligence", donde surge una nueva modalidad organizacional: la red virtual. Concomitantemente, la nueva economía se distingue por estar y ser en Red donde hay una profunda y rica interconexión dentro de las organizaciones.

\section{Ecuador y el cambio en la matriz Productiva}

La matriz productiva es la agrupación de interacciones inherentes entre los diferentes actores que tienen toda sociedad, en la cual se procesan los recursos (renovables y no renovables) íntegramente que posee una nación, con los cuales generan una gama extensa de producción. Estos procesos incluyen los productos y servicios (basados en los gustos y preferencias).

\section{La Globalización Internacional}

La globalización internacional tiene como objetivo primordial, la búsqueda y utilización de capacidades endógenas y exógenas más competitivas en el ámbito mundial para poder generan procesos, productos y servicios que posean la mejor calidad y precios competitivos y, así poder satisfacer las exigencias (gustos y preferencias) cada vez mayores de los mercados locales, regionales y sectores emergentes. Para lo cual la competitividad, es un sentido clave, y por lo tanto una condición sine qua non.

Como tal, implica la capacidad endógena que se deben tener las empresas de un determinado país para competir eficazmente en los mercados internacionales, para que de manera paralela se vea incrementando al mismo tiempo los ingresos reales de la economía.

\section{Las nuevas tendencias que aparecen en el desarrollo mundial se caracterizan, así:}

Globalización, tanto de los mercados y su especialización. En la cual las empresas buscan llegar a todo el mundo y, al mismo tiempo, adaptarse a las condiciones específicas de cada región. Por lo que la producción se ve caracterizada por la flexibilidad, modularidad y adaptabilidad, considerando el tamaño y características de las unidades productivas, así como de las instituciones, los mercados y los servicios locales. 
La interdependencia de la economía mundial. Crecimiento sin precedentes de los flujos de comercio internacional y de capitales, considerando las leyes locales.

La gestión de tecnología e innovación organizacional como elementos claves en la dirección de las empresas. Las corrientes culturales empresariales de excelencia que se ven involucradas las organizaciones que incorporan con nuevos valores, y a su vez promueven el trabajo en equipo, y círculos de calidad, las cuales rompen con los paradigmas de los modelos burocráticos excesivamente jerarquizados en la toma de decisiones eficaces.

Factor humano como talento humano, que causa y objeto del desarrollo y una mayor valoración de la creatividad y la capacidad de innovar y emprender.

Cambio tecnológico y globalización internacional

Como un resultado concreto y especifico de las políticas tecnológicas adoptadas tanto en los países de la OECD como en los del Sudeste Asiático ha sido la creación de redes extensas de cooperación inter-empresarial en I\&D y tecnología a escala internacional

Un conjunto de tendencias indica el rápido proceso de internacionalización de la I\&D industrial y del desarrollo de nuevas tecnologías, tales como:

El aumento considerable de I\&D que se hace en países industrializados por parte de firmas de propiedad extranjeras;

El aumento considerable de las relaciones entre firmas extranjeras y las organizaciones nacionales de investigación;

La escala de cooperación inter-empresarial en 1\&D como transnacional;

La expansión de los intercambios y compartir resultados por compañías de diferentes países.

El termino tecno-globalización se metaforizó para señalar el carácter internacional de la I\&D y la innovación. La internacionalización de la tecnología es, por tanto, parte importante y fundamental de la tendencia actual hacia la globalización de la economía

Para generar un nuevo esquema, se deben cambiar los patrones de especialización de la economía, con lo cual el estado plantea pasar de una economía con especialización primaria exportadora y extractivita a una economía que privilegie la producción con valor agregado y que se haya diversificado, basados en modelos de nuevos conocimientos, lo cual abarca específicamente la innovación, sustentado en la tecnología. 


\section{La Pymes como grupo económico}

La Pymes son un grupo de empresas que por su operatividad emplea en un número menor de 200 personas, por lo general sus procesos son ágil, efectivos y simplificados su objetivo primordial es dinamizar la economía del país, lo que se busca con meta final es la integración de manera inherente para su sustentabilidad en el tiempo, y así poder fortalecer la economía del país

\section{Conclusiones}

Para poder lograr los objetivos, impuesto por el gobierno es importante considerar, que el país necesitas mas centro de incubación de empresas para poder establecer una mejor interacción entre los actores fundamentales en el proceso de una idea de negocios, la creación de programas e incentivos que buscan de que se sustenten y mantengan en el tiempo logrando una estabilidad laboral para las personas que trabajan en dichas empresas.

Otro factor importante que podemos visualizar a nivel mundial es la simplificación de funciones que va acorde a los cambios tecnológicos que tiene como objetivo reducir el proceso.

\section{Referencias Bibliográficas}

Chaverri, P. (2012) Roles, retos y oportunidades de los actores no--- gubernamentales en la atención de lo social: el caso de las Universidades en general y de la Universidad ULACIT en particular

Plan Nacional del Buen Vivir (2013 -2017), Objetivo No. 3 Mejorar la calidad de vida de la población.

Minniti, M. (2012) El emprendimiento y el crecimiento económico de las naciones Economía industrial, No 383, 2012 (Ejemplar dedicado a: Mujer y desafío emprendedor en España), Pp.2330

Plan Nacional del Buen Vivir (2013 -2017), Objetivo No. 4 Fortalecer las capacidades y potencialidades de la ciudadanía

Plan Nacional del Buen Vivir (2013 -2017), Objetivo No. 10 Impulsar la transformación de la matriz productiva

Pineda, K; Márquez, M.; Morales, R., Ortiz, M. (2011). Modelos y mecanismos de interacción universidad-empresa-estado: retos para las universidades colombianas [en línea], Disponible en: 
http://revistas.lasalle.edu.co/index.php/ed/article/view/193.

Ramírez, M. (2010). La Alianza Universidad-Empresa-Estado: una estrategia para promover innovación Pp.112-133.

Velho, L. Velho, P. (2010). Las políticas e instrumentos de vinculación Universidad-Empresa en los países del MERCOSUR. 